\title{
Effective Vortex Mass from Microscopic Theory
}

\author{
Jung Hoon Han, ${ }^{1,2}$ June Seo Kim, ${ }^{1}$ Min Jae Kim, ${ }^{1}$ and Ping $\mathrm{Ao}^{3}$ \\ ${ }^{1}$ Department of Physics, Sung Kyun Kwan University, Suwon 440-746, Korea \\ ${ }^{2}$ CSCMR, Seoul National University, Seoul 151-747, Korea \\ ${ }^{3}$ Department of Mechanical Engineering, University of Washington, Seattle, WA 98195, USA
}

(Dated: November 12, 2018)

\begin{abstract}
We calculate the effective mass of a single quantized vortex in the BCS superconductor at finite temperature. Based on effective action approach, we arrive at the effective mass of a vortex as integral of the spectral function $J(\omega)$ divided by $\omega^{3}$ over frequency. The spectral function is given in terms of the quantum-mechanical transition elements of the gradient of the Hamiltonian between two Bogoliubov-deGennes (BdG) eigenstates. Based on self-consistent numerical diagonalization of the BdG equation we find that the effective mass per unit length of vortex at zero temperature is of order $m\left(k_{f} \xi_{0}\right)^{2}\left(k_{f}=\right.$ Fermi momentum, $\xi_{0}=$ coherence length), essentially equaling the electron mass displaced within the coherence length from the vortex core. Transitions between the core states are responsible for most of the mass. The mass reaches a maximum value at $T \approx 0.5 T_{c}$ and decreases continuously to zero at $T_{c}$.
\end{abstract}

PACS numbers:

Phase coherence is the defining characteristic of the superfluid matter, and vortices - the quantized twist of the underlying phase texture - are the unique elementary excitations of the condensate. Their ubiquity as well as their role in transport and phase transition makes the dynamics of an isolated vortex or their array an integral part of our understanding of superfluidity.

The motion of a single vortex may be phrased as a Newtonian equation, $M_{v}\left(d^{2} \mathbf{r}_{v} / d t^{2}\right)=\mathbf{F}_{v}$, where $\mathbf{F}_{v}$ is the force acting on the vortex, and $M_{v}$ is its effective mass. The position of the vortex is represented by $\mathbf{r}_{v}$. The mass of a vortex is entirely effective in nature [1]: it requires a force to drag a $h / 2 e$ flux quantum across a type-II superconductor by an external magnet (assuming zero mass for the magnet itself) because, in so moving, the vortex interacts with the surrounding electrons. The virtual transitions among the quasiparticle states caused by the vortex motion lead to renormalization of mass, which in this case will account for the entire vortex mass. Real transitions between the states, on the other hand, lead to dissipation.

The Newtonian description of the vortex motion is a limiting case of the more general quantum mechanical formulation. Quantum-mechanical law of motion is most easily derived in the effective action approach 2 . In essence, one divides the dynamics into that of quasiparticles and the vortex, then by integrating out the quasiparticle degrees of freedom, one is left with the effective dynamics of the vortex alone. This way of studying vortex dynamics was first conceived in the work of Simánek $\underline{3}$. and later extensively developed by Ao and Zhu $[\underline{4},[5]$. In this paper, we derive the mass formula from the effective action and calculate it using BCS theory for temperatures $0<T<T_{c}$.

The effective action $S_{\text {eff }}$ of a single vortex of a BCS superconductor, centered at $\mathbf{r}_{v}(\tau)$ at imaginary time $\tau$, is given by $[3,4,[5]$

$$
\frac{1}{8} \int_{0}^{\beta} d \tau \int_{-\infty}^{\infty} d \tau^{\prime} \int_{0}^{\infty} d \omega J(\omega) e^{-\omega\left|\tau-\tau^{\prime}\right|}\left|\mathbf{r}_{v}(\tau)-\mathbf{r}_{v}\left(\tau^{\prime}\right)\right|^{2} .
$$

The spectral function is the quantity

$$
J(\omega)=\left.\sum_{a b} \delta\left(\omega-\left|E_{a}-E_{b}\right|\right)\left|f\left(E_{a}\right)-f\left(E_{b}\right)\right|\left\langle a\left|\frac{\partial H_{0}}{\partial \mathbf{r}_{v}}\right| b\right\rangle\right|^{2} .
$$

Fermi distribution function with energy $x$ is denoted $f(x)$. There is an additional action in $S_{\text {eff }}$ pertaining to the transverse motion of the vortex, which we do not consider here [5]. The states $a, b$ are the eigenstates of the Bogoliubov-deGennes (BdG) Hamiltonian $H_{0}$ in the presence of vortex at $\mathbf{r}_{v}$, with energies $E_{a}$ and $E_{b}$ respectively. When we assume that the dynamics is sufficiently local in time [6] , we may approximate $\mathbf{r}_{v}(\tau)-\mathbf{r}_{v}\left(\tau^{\prime}\right) \approx\left(\tau-\tau^{\prime}\right) \dot{\mathbf{r}}_{v}(\tau)$ and write

$$
S_{e f f} \approx \frac{1}{2} \times\left(\int_{0}^{\infty} d \omega \frac{J(\omega)}{\omega^{3}}\right) \times \int_{0}^{\beta} d \tau\left(\frac{d \mathbf{r}_{v}}{d \tau}\right)^{2} .
$$

The effective vortex mass is the quantity in parenthesis, given by

$$
M_{v}=\sum_{a b}\left|\frac{f\left(E_{a}\right)-f\left(E_{b}\right)}{\left(E_{a}-E_{b}\right)^{3}}\right|\left|\left\langle a\left|\frac{\partial H_{0}}{\partial \mathbf{r}_{v}}\right| b\right\rangle\right|^{2} .
$$

This formula allows an explicit calculation of the vortex mass using the BCS theory of superconductivity at arbitrary temperature.

Conclusions in the past vary regarding the vortex effective mass. One group of theories predicts a "small" mass of roughly one electron mass per atomic length [7], whereas another group predicts a "large" mass, of order 
$m\left(k_{f} \xi_{0}\right)^{2}\left(k_{f}=\right.$ Fermi momentum, $\xi_{0}=$ coherence length at $T=0)$ [ . In either case the theories are limited to zero temperature, and finite-temperature generalization does not seem to be straightforward.

The eigenstates of the BdG equation in the cylindrical coordinates is written in the general form [ $]$

$$
\psi_{a}(\mathbf{r})=\frac{1}{\sqrt{2 \pi L}} e^{i k_{z} z} e^{i \mu \theta}\left(\begin{array}{c}
e^{-i \frac{\theta}{2}} u_{\alpha}(r) \\
e^{+i \frac{\theta}{2}} v_{\alpha}(r)
\end{array}\right)
$$

in the presence of a gap function $\Delta(\mathbf{r})=\Delta(r) e^{-i \theta}$ for a rectilinear vortex of length $L$ centered at $r=0$. The radial functions $\left(u_{\alpha}(r), v_{\alpha}(r)\right)$ are obtained as eigenfunctions of the coupled differential equation ( $r$ dependence in $u, v$ and $\Delta$ is implicit)

$$
\begin{aligned}
& r^{2} u^{\prime \prime}+r u^{\prime}+\left[\left(k_{r}^{2}+2 m E\right) r^{2}-\left(\mu-\frac{1}{2}\right)^{2}\right] u=2 m r^{2} \Delta v \\
& r^{2} v^{\prime \prime}+r v^{\prime}+\left[\left(k_{r}^{2}-2 m E\right) r^{2}-\left(\mu+\frac{1}{2}\right)^{2}\right] v=-2 m r^{2} \Delta(6)
\end{aligned}
$$

where $k_{r}^{2}=k_{f}^{2}-k_{z}^{2}$. Self-consistency requires that the $r$-dependent gap function obey the relation $\Delta(r)=$ $V \sum_{\alpha} u_{\alpha}(r) v_{\alpha}(r)\left[1-2 f\left(E_{\alpha}\right)\right]$ for some choice of the pairing interaction strength $V$. The eigenstates are labeled by $k_{z}, \mu$, and $\alpha$, which labels a set of energy levels for a given choice of $\left(k_{z}, \mu\right)$. Uniqueness of the wavefunction requires that $\mu$ be half-odd integers. Using these eigenstates, the transition amplitude needed in evaluating Eq. (4) can be written out in the form $-\left\langle\alpha\left|\partial H_{0} / \partial \mathbf{r}_{v}\right| \beta\right\rangle=$ $(\hat{x} \mp i \hat{y} / 2) \times$

$$
\int_{0}^{R}\left\{\left(u_{\alpha} v_{\beta}+u_{\beta} v_{\alpha}\right) r \frac{d \Delta}{d r} \pm\left(u_{\alpha} v_{\beta}-u_{\beta} v_{\alpha}\right) \Delta\right\} d r
$$

Upper and lower signs correspond to $\mu_{\alpha}=\mu_{\beta} \pm 1$. The two eigenstates differ by one unit of angular momentum due to the gradient operator which connects them. By integrating by parts, Eq. (7) is equal to (assuming $\mu_{\alpha}=$ $\left.\mu_{\beta}+1\right)$

$$
\left(E_{\beta}-E_{\alpha}\right) \int_{0}^{R}\left\{\left(u_{\alpha} \frac{d u_{\beta}}{d r}+v_{\alpha} \frac{d v_{\beta}}{d r}\right) r-\left(\left(\mu_{\beta}-\frac{1}{2}\right) u_{\alpha} u_{\beta}+\left(\mu_{\beta}+\frac{1}{2}\right) v_{\alpha} v_{\beta}\right)\right\} d r+\frac{R}{2 m}\left(\frac{d v_{\alpha}}{d r} \frac{d v_{\beta}}{d r}-\frac{d u_{\alpha}}{d r} \frac{d u_{\beta}}{d r}\right)_{r=R} .
$$

We assume a hard-wall boundary condition at $r=R$. One must be careful in treating the boundary term at $r=R$ on the r.h.s. of the above equation, which is generally non-zero.

We follow earlier works 10 and write the eigenfunctions in the form

$$
\begin{gathered}
u_{\alpha}(r)=\sum_{i} c_{\alpha i} \phi_{n i}(r), \quad v_{\alpha}(r)=\sum_{i} d_{\alpha i} \phi_{n+1, i}(r), \\
\phi_{n i}(r)=\frac{\sqrt{2}}{R J_{n+1}\left(z_{n i}\right)} J_{n}\left(z_{n i} \frac{r}{R}\right) .
\end{gathered}
$$

$z_{n i}$ is the $i$-th zero of the Bessel function $J_{n}$. Integer value $n$ is related to the angular momentum by $\mu_{\alpha}=n+\frac{1}{2}$. Coefficients $\left(c_{\alpha i}, d_{\alpha i}\right)$ are determined from matrix diagonalization. Negative- $\mu$ states need not be considered separately, instead one can use a positive- $\mu$ eigenstate $\left(u_{\alpha}, v_{\alpha}\right)$ of energy $E_{\alpha}$ to construct a negative- $\mu$ eigenstate, given by $\left(v_{\alpha},-u_{\alpha}\right)$, of opposite energy $-E_{\alpha}$.

With the eigenfunctions thus obtained, we evaluate the vortex mass using Eq. (4), and the transition amplitude given by Eq. (8). Furthermore, Eq. (8) can be re-expressed using the coefficients $\left(c_{\alpha i}, d_{\alpha i}\right)$ and making use of Bessel identities. For $\mu_{\alpha}=n+\frac{1}{2}, \mu_{\beta}=n-\frac{1}{2}$, $n>0$, Eq. [8] becomes

$$
A_{n, \alpha \beta}=\frac{2}{R} \sum_{i j} c_{\alpha i} c_{\beta j} z_{n i} z_{n-1, j}\left(\frac{E_{\alpha}-E_{\beta}}{z_{n i}^{2}-z_{n-1, j}^{2}}-\frac{\xi_{0}}{R^{2}}\right)+\frac{2}{R} \sum_{i j} d_{\alpha i} d_{\beta j} z_{n+1, i} z_{n j}\left(\frac{E_{\alpha}-E_{\beta}}{z_{n+1, i}^{2}-z_{n j}^{2}}+\frac{\xi_{0}}{R^{2}}\right),
$$

and for $\mu_{\alpha}=+\frac{1}{2}, \mu_{\beta}=-\frac{1}{2}$, it equals

$$
B_{\alpha \beta}=\frac{2}{R} \sum_{i j} c_{\alpha i} d_{\beta j} z_{0 i} z_{1 j} \frac{J_{0}\left(z_{1 j}\right)}{J_{2}\left(z_{1 j}\right)}\left(\frac{E_{\alpha}+E_{\beta}}{z_{0 i}^{2}-z_{1 j}^{2}}-\frac{\xi_{0}}{R^{2}}\right)+\frac{2}{R} \sum_{i j} d_{\alpha i} c_{\beta j} z_{1 i} z_{0 j} \frac{J_{0}\left(z_{1 i}\right)}{J_{2}\left(z_{1 i}\right)}\left(\frac{E_{\alpha}+E_{\beta}}{z_{0 j}^{2}-z_{i 1}^{2}}-\frac{\xi_{0}}{R^{2}}\right) .
$$

In deriving Eqs. (10)-(11) we expressed energy in units 
the core, denoted $\Delta(0)$, and the length in units of $k_{f}^{-1}$. In Eq. (10), $\alpha$ and $\beta$ refer to eigenstates with a given angular momenta $\mu_{\alpha}$ and $\mu_{\beta}$, each. We made use of the mapping between negative- $\mu$ and positive- $\mu$ eigenstates to express the result in Eq. (11) solely in terms of the $\mu=+\frac{1}{2}$ eigenstates. The vortex mass can be calculated using $M_{v}=$

$$
\begin{aligned}
& 2 m \cdot \frac{\xi_{0}}{2} \cdot 2 \sum_{n>0, \alpha \beta}\left|\frac{f\left(E_{\alpha}\right)-f\left(E_{\beta}\right)}{\left(E_{\alpha}-E_{\beta}\right)^{3}}\right| A_{n, \alpha \beta}^{2} \\
+ & 2 m \cdot \frac{\xi_{0}}{2} \sum_{n=0, \alpha \beta}\left|\frac{f\left(E_{\alpha}\right)-f\left(-E_{\beta}\right)}{\left(E_{\alpha}+E_{\beta}\right)^{3}}\right| B_{\alpha \beta}^{2} .
\end{aligned}
$$

Factor 2 multiplying the first term reflects the negative- $\mu$ contributions which equals those of the positive- $\mu$ transitions.

Equations (10)-12) complete the formal derivation of the vortex mass formula in the superconductor. Using Eqs. (10)-(11) gives dramatic improvement in both the time and accuracy of the calculation of the transition amplitude over the brute-force numerical integration of Eq. (7) or (8).

In the following we discuss the vortex mass calculated using Eq. (12), with the eigenstates obtained from selfconsistent numerical diagonalization of Eq. (6). We choose the coherence length $E_{f} / \Delta(0) \equiv \xi_{0}=20$ and the radius of the boundary $R=100$. For each angular momentum $\mu$, eigenstates with energies within $\pm 10 \Delta(0)$ were retained. This left us with about 90 eigenstates for $\mu=1 / 2$, and a decreasing number of states for larger $\mu$. Calculations were restricted to $k_{z}=0$ only. Varying $k_{z}$ will lead to different effective Fermi momentum $k_{r}=\sqrt{k_{f}^{2}-k_{z}^{2}}$ in Eq. (6) and will not change the qualitative conclusion from the $k_{z}=0$ case. The energy gap vanishes completely at $T \approx 0.571 \Delta(0)$, which we take as the transition temperature $T_{c}$. We work at several (reduced) temperatures $t \equiv T / T_{c}$ in the range $0<t<1$.

Figure 1 shows the calculated gap at large distance from the core, $\Delta(T)$, and the $\mu=+\frac{1}{2}$ core energy level, $\epsilon_{\frac{1}{2}}(T)$, for the temperatures we considered. Both quantities decrease monotonically with $T$. For $T$ sufficiently close to $T_{c}$ we can no longer resolve the core levels as distinct from the continuum.

Referring to Eq. (12) we may divide the mass as arising from transitions between the core states $\left(M_{v}^{c c}\right)$, coreto-extended states $\left(M_{v}^{c e}\right)$, and finally between extended states $\left(M_{v}^{e e}\right)$, as $M_{v}=M_{v}^{c c}+M_{v}^{c e}+M_{v}^{e e}$. As it turns out the matrix element, Eq. (7), is vanishingly small between a core and an extended state, and the mass is effectively $M_{v} \approx M_{v}^{c c}+M_{v}^{e e}$. In Fig. 2(a) we show the total mass $M_{v}(T)$ and the core-to-core mass, $M_{v}^{c c}(T)$. As is evident from the figure, $M_{v}^{c c}(T) \gg M_{v}^{e e}(T)$ for all temperatures except very near $T_{c}$ where core levels are not resolved, but here the total mass is vanishingly small anyway. Both the total mass $M_{v}$ and the level-resolved

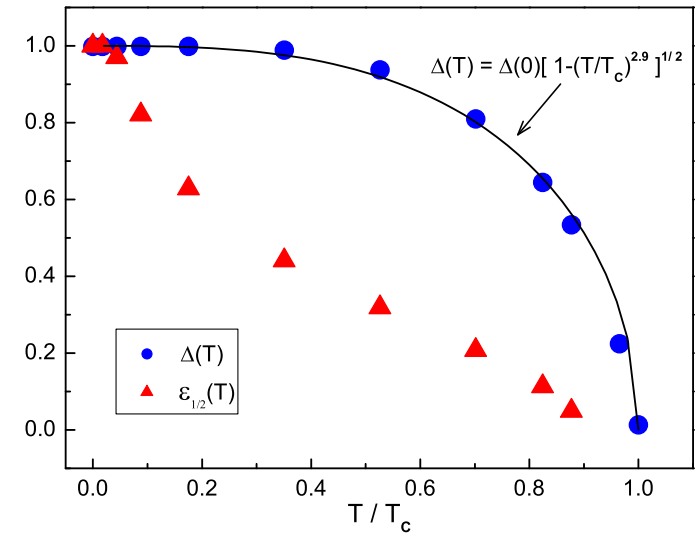

FIG. 1: Pair potential well away from the core, $\Delta(T)$ (blue), and the core energy level for $\mu=+\frac{1}{2}, \epsilon_{1 / 2}(T)$ (red), plotted vs. $T / T_{c}$. Both quantities are normalized by their respective $T=0$ values, with $\epsilon_{1 / 2}(0) / \Delta(0)=0.088$ from self-consistent calculation. $\Delta(T)$ shows excellent fit to $\Delta(0) \sqrt{1-\left(T / T_{c}\right)^{2.9}}$ (black curve).

masses $M_{v}^{c c}$ and $M_{v}^{e e}$ reach a maximum value around $T=0.5 T_{c}$.

The core-level contribution to mass can be further grouped according to the angular momentum channels $\left(\mu_{\alpha}=n+\frac{1}{2}\right) \leftrightarrow\left(\mu_{\beta}=n-\frac{1}{2}\right)$ over which the transition takes place. We denote such angular-momentumresolved, core-to-core mass, $M_{v}^{c c, n}(T)$. Figure 2(b) shows $M_{v}^{c c, 0}(T)$ and $\sum_{n \neq 0} M_{v}^{c c, n}(T)=M_{v}^{c c}(T)-M_{v}^{c c, 0}(T)$. We find that $M_{v}^{c c, 0}(T)$, between $\mu= \pm \frac{1}{2}$ core levels, survives at zero temperature and monotonically decreases at higher $T$. The $n \neq 0$ channels give zero mass for $T=0$, reaches a maximum at some intermediate $T$, and decreases to zero at $T=T_{c}$. This behavior accounts for the observed maximum in the mass $M_{v}(T)$.

The results shown in Fig. 2 (b) can be nicely understood thanks to the approximate identity that holds for the core levels [8] , which states $\left|\left\langle\alpha\left|\nabla_{v} H_{0}\right| \beta\right\rangle\right| \approx\left|E_{\alpha}-E_{\beta}\right|$. We have verified that this relation holds with excellent accuracy for all temperatures, except when $T \approx T_{c}$. Then, we can approximate (see Eq. (12))

$$
M_{v}^{c c, n}(T) \approx 2 m \xi_{0}\left|\frac{f\left(\epsilon_{n-\frac{1}{2}}(T)\right)-f\left(\epsilon_{n+\frac{1}{2}}(T)\right)}{\epsilon_{n-\frac{1}{2}}(T)-\epsilon_{n+\frac{1}{2}}(T)}\right| .
$$

In particular, for $n=0$ we have the mass $M_{v}^{c c, 0}(T)=$ $2 m \xi_{0} \tanh \left(\beta \epsilon_{\frac{1}{2}}(T)\right) / 2 \epsilon_{\frac{1}{2}}(T)$. At zero temperature we get $M_{v}^{c c, 0}(0)=2 m \xi_{0} / 2 \epsilon_{\frac{1}{2}}(0) \sim m \xi_{0}^{2}$ since $\Delta(0) / \epsilon_{\frac{1}{2}}(0) \sim \xi_{0}$. This is precisely the mass of the electrons occupying the area $\sim \xi_{0}^{2}$. Using the $\epsilon_{\frac{1}{2}}(T)$ values shown in Fig. 1] we find that the approximate formula in Eq. (13) for $n=$ 0 reproduces $M_{v}^{c c, 0}(T)$ obtained from the more general 

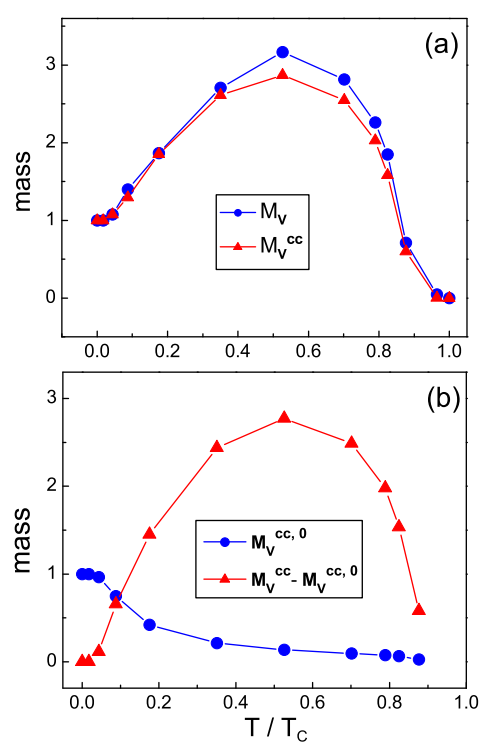

FIG. 2: (a) Effective mass $M_{v}(T)$ and the core-to-core contribution to mass $M_{v}^{c c}(T)$ at temperature $T / T_{c}$, normalized by $M_{v}(0)$. One finds $M_{v}(T) \approx M_{v}^{c c}(T)$ over most of $T$. (b) $M_{v}^{c c}(T)=M_{v}^{c c, 0}(T)+\sum_{n \neq 0} M_{v}^{c c, n}(T)$ (see text for definition). $M_{v}^{c c, 0}(T)$ and $M_{v}^{c c}(T)-M_{v}^{c c, 0}(T)$ are plotted separately. $M_{v}^{c c, 0}(T)$ is monotonically decreasing while the higher$n$ channels give a maximum mass at $T / T_{c} \approx 0.5$. The core levels are not well resolved for $T$ too close to $T_{c}$, which explains the absence of data points for $T \approx T_{c}$.

formula, Eq. (12), with very high accuracy.

On the other hand, $M_{v}^{c c, n \neq 0}(T) \approx$ $\beta /\left(4 \cosh ^{2}\left(\beta \epsilon_{n}(T) / 2\right)\right), \epsilon_{n}(T) \equiv\left(\epsilon_{n+\frac{1}{2}}(T)+\epsilon_{n-\frac{1}{2}}(T)\right) / 2$, provided the temperature is much larger than the typical core energy spacing, $T \gg\left|\epsilon_{n+\frac{1}{2}}(T)-\epsilon_{n-\frac{1}{2}}(T)\right|$. As the typical core energy spacings are a few percent of the energy gap $\Delta(T)$, this is not a very restrictive condition except near $T=0$. Then one can easily check that this approximate form for $M_{v}^{c c, n}(T)$ rises to a maximum value for $T \approx 0.69 \epsilon_{n}$ provided $\epsilon_{n}$ is treated as temperature-independent. Therefore, the maximum in the mass shown in Fig. 2(a) is due to the core-to-core transitions in the higher angular momentum channels, $\sum_{n \neq 0} M_{v}^{c c, n}(T)$.

Summarizing our findings, (i) At zero temperature the effective mass $M_{v}(T=0)$ is the mass of the electrons forming a cylinder of radius $\xi_{0}$. It increases upon higher temperature, reaching a maximum at $T \approx 0.5 T_{c}$, and vanishes at $T_{c}$. (ii) The transition between the localized eigenstates forming the core spectrum is mainly responsible for the effective mass. Hence, $M_{v}(T) \approx M_{v}^{c c}(T)$. On the other hand, the dissipation experienced by the moving vortex is due to the extended states as it requires transition between states of the same energy (See Refs.
[4, 5] for a discussion of vortex friction).

Suppose now that the core levels got smeared due to the impurities. If all higher angular momentum core levels except $n=0$ disappeared, we would expect $M_{v}^{c c, n \neq 0}(T)$ is effectively zero, and the mass behaves as $M_{v}(T) \approx M_{v}^{c c, 0}(T)$ showing the monotonic behavior of Fig. 2(b). If all core levels disappeared, we will be left with the extended states contributions which are shown to be quite small. It is conceivable that in this case the mass becomes "small", in agreement with predictions of Ref. 7].

In closing we mention that studying the size $(R)$ dependence may be necessary to get a better estimate of the extended-states mass, $M_{v}^{e e}(R)$. Since the core states are localized within a few coherence lengths from the origin, our choice $R=10 \xi_{0}$ is presumably adequate to conclude $M_{v}^{c c}(R=100) \approx M_{v}^{c c}(R=\infty)$. Extended states do not meet this requirement, at least for ultra-clean systems with no impurity scattering to provide the cutoff. The question of friction 4, 5, 11], which involves transitions between same-energy extended states, will also require the study of $R$-dependence of the transition rates. We will return to these issues in a future publication.

HJH was supported by grant No. R01-2002-000-003260 from the Basic Research Program of the Korea Science \& Engineering Foundation. We thank helpful conversation with X.-M. Zhu.

[1] A nice account of the effective mass arising from interaction effects in elementary particles is given by $\mathrm{F}$. Wilczek in Physics Today, 52 (November), 11 (1999) and 53 (January), 13 (2000).

[2] A. O. Caldeira and A. J. Leggett, Ann. Phys. (NY) 149, 374 (1983).

[3] E. Šmánek, Phys. Lett. A 194, 323 (1994).

[4] P. Ao and X.-M. Zhu, Physica C 282-287, 367 (1997).

[5] P. Ao and X.-M. Zhu, Phys. Rev. B 60, 6850 (1999).

[6] This will be the case when the vortex is moving sufficiently slowly, or if it's moving along a straight line.

[7] H. Suhl, Phys. Rev. Lett. 14, 226 (1965); J.-M. Duan and A. J. Leggett, Phys. Rev. Lett. 68, 1216 (1992); J.M. Duan, Phys. Rev. B 48, 333 (1993).

[8] N. B. Kopnin and M. M. Salomaa, Phys. Rev. B 44, 9667 (1991); T. C. Hsu, Physica C 213, 305 (1993); D. M. Gaitonde and T. V. Ramakrishnan, Phys. Rev. B 56, 11951 (1997); N. B. Kopnin and V. M. Vinokur, Phys. Rev. Lett. 81, 3952 (1998).

[9] P. G. de Gennes, Superconductivy of Metals and Alloys (Addison-Wesley, 1989).

[10] F. Gygi and M. Schlüter, Phys. Rev. B 41, 822 (1990); ibid. 43, 7609 (1991); N. Hayashi, T. Isoshima, M. Ichioka, and K. Machida, Phys. Rev. Lett. 80, 2921 (1998).

[11] J. Bardeen and M. J. Stephen, Phys. Rev. 140, A1197 (1965). 